\title{
Calendula officinalis/Plantago major/Cochlearia armoracia/Hamamelis virginiana Herbal Toothpaste
}

National Cancer Institute

\section{Source}

National Cancer Institute. Calendula officinalis/Plantago major/Cochlearia

armoracia/Hamamelis virginiana Herbal Toothpaste. NCI Thesaurus. Code C90561.

A phytochemical-based toothpaste containing Calendula officinalis, Plantago major, Cochlearia armoracia, Hamamelis virg iniana with potential soothing activity. Calendula officinalis/Plantago major/Cochlearia armoracia/ Hamamelis virg iniana herbal toothpaste may relieve the discomfort associated with radiation-induced mucositis. 